\section{Salud, desarrollo humano y gobernabilidad en América Latina y el Caribe a inicios del siglo XXI}

Juan Antonio Casas-Zamora ${ }^{1}$

Palabras clave: pobreza, crecimiento económico, servicios de salud, gobernabilidad, Américas.

\footnotetext{
División de Salud y Desarrollo Humano (HDP), Organización Panamericana de la Salud. La correspondencia debe enviarse a Juan Antonio Casas-Zamora, PAHO/WHO (HDP), 525, 23rd St., NW, Washington, D.C. 20037, Estados Unidos de América. Correo electrónico: casasjua@paho.org
}

\section{DESARROLLO HUMANO SOSTENIBLE Y SALUD}

El desarrollo humano es el proceso de aumentar las opciones de las personas, lo cual se logra ampliando las capacidades esenciales y de funcionamiento. Las tres capacidades esenciales para el desarrollo humano son que las personas tengan una vida larga y sana, que posean los conocimientos necesarios y que tengan acceso a los recursos necesarios para un nivel de vida aceptable (1). Las inquietudes con respecto a la equidad ocupan el primer plano en la perspectiva del desarrollo humano, en particular la equidad en las capacidades y las oportunidades básicas para todos, es decir: la equidad en el acceso a la educación, en la salud y en los derechos políticos. La esencia y la prueba de las estrategias de desarrollo humano sostenibles deben ser alcanzar una forma de vida sostenible para todos. Estas estrategias son necesarias para el desarrollo centrado en las personas, con inquietudes por el empoderamiento humano, la participación, la igualdad de género, el crecimiento equitativo, la reducción de la pobreza y la sostenibilidad a largo plazo.

Las disparidades económicas y sociales prevalecientes en la Región, y en particular las de salud, deben representar un objeto de fundamental preocupación intersectorial, que el conjunto de nuestras sociedades se encuentra compelido a enfrentar de manera concertada con políticas de impacto nacional y regional. Sin embargo, por razones técnicas y políticas, a nivel macro no ha resultado sencillo establecer de forma clara las relaciones causales que determinan estas inequidades $\mathrm{y}$, sobre todo, ponderar o medir adecuadamente el nivel y la naturaleza de las interacciones entre la salud y el desarrollo económico, político y social. Este vacío tiene repercusiones muy concretas en el campo de la formulación de políticas que busquen reducir las profundas disparidades en salud existentes en la Región.

En los últimos años se ha establecido un mayor consenso alrededor de estas interacciones. Una de las principales consecuencias de este consenso se puede constatar a través del incremento del número de investigaciones destinadas a medir y definir el concepto de equidad en salud (2). En el contexto de los procesos de globalización, la coexistencia del crecimiento económico con un incremento de las inequidades es un rasgo característico del desarrollo reciente en la mayoría de los países de la Región; las disparidades en salud injustas, evitables y resultan- 
tes de la falta de opción de los afectados son calificadas como inequidades en salud (3).

El estudio de las inequidades ha develado en buena medida las importantes diferencias que se esconden detrás de muchos indicadores medios. Las perspectivas de género y de etnia han generado un número creciente de evidencias que se suman a los estudios sobre salud y capital social, pobreza, clase social y diferencias de ingresos (4). Otras perspectivas, como el estudio de la condición social en poblaciones migrantes, los fenómenos de urbanización asimétrica (5) y la distribución de ingresos por grupos etarios, significativos en un contexto de envejecimiento de la población (6), comienzan a sumar nuevas evidencias para la formación de políticas orientadas a la equidad. En muchos casos se verifica que la apropiación de los beneficios del desarrollo tecnológico, del crecimiento económico y de la misma globalización tiende a concentrarse en ciertos grupos sociales, ampliando las disparidades $\mathrm{y}$, en sus formas extremas, contribuyendo a poner en riesgo la gobernabilidad democrática.

La región de América Latina y el Caribe (ALC) muestra las mayores disparidades en ingresos, así como en otros factores socioeconómicos que determinan la salud, y estas disparidades han ido en aumento desde 1980 (7). Aunque la salud general ha mejorado en los países de ALC, no se han obtenido iguales ganancias en la situación de salud de los diversos grupos socioeconómicos. El mejoramiento de la salud general parece inclinarse de manera desproporcionada hacia aquellos que ya gozan de mayores ventajas sociales y económicas en la sociedad, mientras que la salud de los grupos desfavorecidos mejora menos sistemáticamente y a un ritmo mucho más moderado (8).

\section{LA SALUD EN RELACIÓN CON EL CRECIMIENTO ECONÓMICO}

"La correlación positiva entre la salud y los ingresos per cápita es una de las relaciones mejor conocidas en el desarrollo internacional" (9). El nivel de salud de la población, particularmente durante períodos largos, tiende a asociarse con el nivel del crecimiento económico y la disponibilidad general de recursos, como se evidencia al correlacionar un indicador de salud como la tasa de mortalidad infantil (TMI) con los ingresos (producto interno bruto: PIB) per cápita, como se muestra en la figura 1 para el caso de los países de ALC. La curva muestra que cuanto mayores son los ingresos, menor es la TMI. La forma cóncava de la curva de ajuste indica que la ganancia en salud por unidad de incremento del ingreso es proporcionalmente mayor en los países, y por extensión en los grupos sociales, más pobres. Por otro lado, la figura 1 muestra un hecho aún más interesante: al comparar las curvas correspondientes a 1995 y 1999 se constata que la TMI media con determinado nivel de ingresos, expresado en valor constante, se redujo

FIGURA 1. Tasa de mortalidad infantil (TMI) y producto interno bruto (PIB) per cápita (en dólares estadounidenses de 1999 ajustados para la paridad de poder adquisitivo). Veintiún países de América Latina y el Caribe, 1995 y 1999

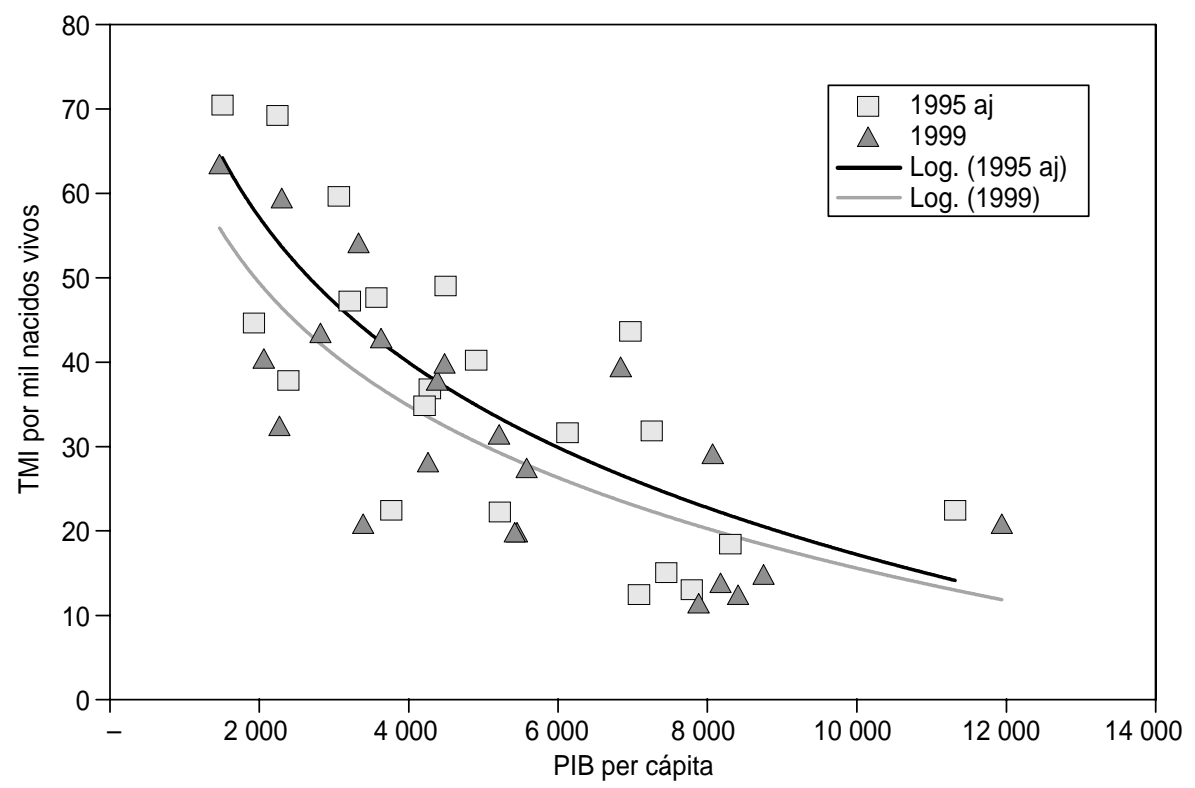


en aproximadamente un $10 \%$ en el período en cuestión, sobre todo en los rangos de menor ingreso; es decir, una parte significativa de la reducción de la TMI de los países de la Región no sería atribuible a cambios de los ingresos, sino a otros determinantes, como la difusión de la información y la aplicación de políticas públicas y sanitarias conducentes a la mejoría de la salud de la población. Esto significa que, aun sin un crecimiento económico real, la situación sanitaria puede mejorar de manera mensurable y que esta tendencia parece distribuida por igual entre todos los niveles de ingresos.

Los países y grupos sociales de mayores ingresos generalmente tienen mejor situación de salud y mejores condiciones generales de vida, ya que tienen más recursos económicos y tecnológicos para satisfacer sus necesidades básicas. Los contextos políticos también son importantes determinantes de la salud. Con pocas excepciones, aquellos países que han desarrollado instituciones de gobierno democráticas y sociedades civiles fuertes, por lo general, han establecido políticas sociales a largo plazo inclinadas a una distribución más amplia de los ingresos y beneficios sociales. Es interesante ver que los países que aplicaron políticas sociales que brindan a sus poblaciones mejor acceso a la educación, a los servicios básicos de salud, a la nutrición y al saneamiento básico han logrado tasas de mortalidad bajas comparadas con los países de igual o aun mejor desempeño económico donde persisten las grandes disparidades de ingresos y recursos $(10,11)$.

En otras palabras, aun en ausencia de un proceso de crecimiento económico, hay países que han logrado invertir en la salud de sus pueblos y alcanzar importantes avances en el bienestar general. Por otro lado, hay pruebas crecientes de que esta inversión en salud es, a su vez, un insumo importante, y hasta indispensable, para alcanzar el crecimiento económico sostenible a largo plazo. Se han identificado varios mecanismos directamente vinculados al impacto positivo de la inversión en salud en el proceso de crecimiento económico:

- Empoderamiento de las mujeres, salud materna, desarrollo del niño en la primera infancia y su productividad futura. Una de las vías más importantes es la mediada por el papel activo de las mujeres en el desarrollo humano. Invirtiendo en la salud de las madres no solo se mejora la nutrición de los niños al nacer, sino también sus logros educacionales posteriores, influyendo así en su futura elección ocupacional y su productividad (12-15).

- Formación de capital humano, salud, nutrición y salarios. La buena salud y nutrición mejoran la productividad de los trabajadores (16). La salud repercute de manera directa sobre los ingresos y la riqueza doméstica, la productividad en el trabajo, la participación de la fuerza laboral, las tasas de ahorro e inversión, los factores demográficos y otros factores de capital humano.

- La repercusión de enfermedades específicas en los resultados económicos. Hay muchos ejemplos de evidente vinculación entre el control o la reducción de enfermedades específicas y su repercusión en la productividad en el trabajo o el aumento de los ingresos. De particular relevancia es el caso del VIH/sida y la malaria (17).

- Repercusión de la morbilidad individual y doméstica en los salarios. El Banco Interamericano de Desarrollo (BID) en conjunto con la Organización Panamericana de la Salud (OPS) coordinaron estudios recientes de encuestas domiciliarias latinoamericanas en un esfuerzo por determinar la relación entre morbilidad y salarios (18). Se encontró que los efectos potenciales de mejorar la salud sobre los salarios individuales son significativos, pero son especialmente grandes entre aquellos con menos capital humano y, en consecuencia, con menores ingresos.

- Repercusiones demográficas. La salud y las variables demográficas desempeñan una función sumamente importante para determinar las tasas de crecimiento económico. Se estima que un aumento del $1 \%$ en la esperanza de vida representa una aceleración del crecimiento del PIB per cápita de más del $3 \%$ anual durante el siguiente cuarto de siglo, y la reducción de la tasa de fecundidad en dos niños por mujer parece ser causa de una aceleración del crecimiento anual en un 1\% (19). Por último, esta relación causal entre la salud y el crecimiento económico se ha constatado mediante estudios macroecónomicos propiciados por la OPS en la Región (20). La medición del impacto de la inversión en salud sobre el crecimiento y la distribución económica ha llevado al hallazgo empírico de que la salud se correlaciona de forma positiva con el crecimiento económico a largo plazo, en un grado que oscila entre un $0,8 \mathrm{y}$ un $1,5 \%$ de crecimiento anual, y de que el efecto máximo se expresa 15 a 20 años después de la mejoría de la situación de salud, lo cual refleja seguramente la naturaleza intergeneracional de la formación de capital humano educativo y sanitario.

\section{POBREZA, INGRESOS Y SALUD}

Pocos temas han sido referidos con tanta frecuencia como las relaciones recíprocas entre la pobreza y la salud. En este sentido, es posible imaginar que resulta necesario reevaluar estas relaciones a inicios del siglo XXI, tomando en cuenta aspectos relativamente más recientes, como los 
estudios sobre la heterogeneidad de la pobreza, la pobreza estructural y la pobreza por ingresos. La pobreza es básicamente la privación o reducción de la capacidad (21). Hay privaciones que son intrínsecamente importantes, como la mala salud, la desnutrición o el analfabetismo, mientras que otras desempeñan un papel instrumental determinante, como los bajos ingresos. El estado de salud de una persona o una población es al mismo tiempo un rasgo constitutivo y un factor decisivo con respecto al desarrollo humano. La mala salud es en sí misma una causa de pobreza, en el sentido de que los miembros menos afortunados de la sociedad son sistemáticamente privados de un componente intrínsecamente necesario de la capacidad óptima, al padecer niveles más altos de mortalidad y morbilidad. Los países en desarrollo que han invertido para proporcionar a sus ciudadanos el acceso universal a los servicios básicos de salud, la seguridad alimentaria y la nutrición, así como a los servicios de agua potable y saneamiento y oportunidades educativas adecuadas, pueden tener poblaciones con ingresos relativamente bajos, pero con un mayor nivel de capacidad y de estado de salud, en comparación con países más ricos, pero con una distribución mucho más desigual de las oportunidades y las capacidades.

Para lograr un desarrollo humano sostenible es vital superar los mecanismos de reproducción de la pobreza, a fin de no enajenar las posibilidades de las futuras generaciones, mediante la operacionalización de estrategias conjuntas y combinadas con todos los sectores, en las que concurran las intervenciones de salud, educación, nutrición y otras necesidades básicas. Una forma clásica y extendida en este sentido es considerar en situación de pobreza a todas las personas que viven con un ingreso diario medio inferior a 2 dólares estadounidenses (USD)
(22). Pero hay formas más precisas de definir la pobreza, que además corrigen el poder adquisitivo de cada moneda, como los estudios basados en el valor de la canasta o cesta alimentaria, entendiendo que las familias que gastan más del $50 \%$ de sus ingresos en ella se encuentran en situación de pobreza, y que las que utilizan todos sus ingresos se encuentran en situación de indigencia, ya que son familias que tienen amenazada su propia subsistencia.

La pobreza afecta a un elevado porcentaje de familias en ALC, estimándose en unos 150 millones de personas. Las cifras en números absolutos siguen aumentando desde 1985, si se considera el criterio de ingreso inferior a USD 2. Si se utilizan indicadores más refinados, no sería exagerado afirmar que, de una forma $u$ otra, la pobreza afecta a la mitad de la población de la Región (23). Llaman la atención los fenómenos asociados, como el de los nuevos pobres (fenómeno que expresa la situación de países que han visto un súbito empobrecimiento de sus capas medias), que se acompaña muchas veces de fenómenos de ruptura y trastorno de las estructuras comunitarias y familiares, con el riesgo de generar impactos negativos a largo plazo para la acumulación de capital humano y social.

El examen de las características asociadas a la pobreza indica que 7 de cada 10 hogares urbanos pobres se encuentran en esa condición debido a los bajos ingresos laborales, 2 a consecuencia del desempleo y 1 preponderantemente debido al número de hijos (cuadro 1) (24). El peso relativo de estas características no ha variado en la década.

El acceso a la educación, junto con la salud, es el instrumento fundamental de acumulación de capital humano y la carencia de ambos componentes del desarrollo humano constituye el principal determinante inmediato de la reproducción intergeneracional de la pobreza. En el área de instrucción

CUADRO 1. Porcentaje de familias pobres en zonas urbanas y distribución por causa principal. Doce países de América Latina, alrededor de 1997

\begin{tabular}{lccccc}
\hline & Índice de pobreza & \multicolumn{4}{c}{ Causa principal (\%) } \\
\cline { 3 - 6 } \multicolumn{1}{c}{ País } & $(\%)$ & Bajos ingresos & Desempleo & Familia numerosa & Otras \\
\hline Argentina (Buenos Aires) & 13 & 42 & 33 & 21 & 4 \\
Bolivia & 47 & 72 & 8 & 11 & 9 \\
Brasil & 25 & 69 & 16 & 11 & 8 \\
Chile & 19 & 58 & 18 & 10 & 13 \\
Colombia (Bogotá) & 30 & 70 & 15 & 10 & 7 \\
Ecuador & 35 & 74 & 9 & 11 & 9 \\
Honduras & 67 & 83 & 7 & 13 & 6 \\
México & 38 & 57 & 10 & 10 & 2 \\
Panamá & 25 & 61 & 20 & 9 & 9 \\
Paraguay (Asunción) & 34 & 67 & 17 & 10 & 4 \\
Uruguay & 6 & 68 & & 6 \\
Venezuela & 42 & &
\end{tabular}

Fuente: Referencia 24. 
CUADRO 2. Desigualdades de los logros educativos en el ciclo básico, por quartiles (primero y cuarto) de ingresos. Zonas urbanas de 12 países de América Latina, 2000

\begin{tabular}{|c|c|c|c|c|c|}
\hline \multirow[b]{2}{*}{ País } & \multirow[b]{2}{*}{$\begin{array}{c}\text { Cuartil } \\
\text { de ingresos }\end{array}$} & \multicolumn{4}{|c|}{$\%$ de niños en edad escolar } \\
\hline & & $\begin{array}{l}\text { Con rezago para } \\
\text { ingresar a la } \\
\text { Educación Básica }\end{array}$ & $\begin{array}{l}\text { Que repiten dos de } \\
\text { los primeros grados }\end{array}$ & $\begin{array}{l}\text { Que interrumpen o se } \\
\text { rezagan en los cuatro } \\
\text { primeros grados }\end{array}$ & $\begin{array}{l}\text { Que interrumpen o se } \\
\text { rezagan en la } \\
\text { educación primaria }\end{array}$ \\
\hline \multirow[t]{2}{*}{ Brasil } & 1 & 8 & 44 & 48 & 67 \\
\hline & 4 & 1 & 5 & 5 & 13 \\
\hline \multirow[t]{2}{*}{ Chile } & 1 & 2 & 14 & 8 & 14 \\
\hline & 4 & 0 & 4 & 2 & 2 \\
\hline \multirow[t]{2}{*}{ Colombia } & 1 & 8 & 21 & 23 & 35 \\
\hline & 4 & 2 & 7 & 4 & 12 \\
\hline \multirow[t]{2}{*}{ Costa Rica } & 1 & 3 & 30 & 17 & 23 \\
\hline & 4 & 0 & 3 & 3 & 4 \\
\hline \multirow[t]{2}{*}{ El Salvador } & 1 & 9 & 19 & 25 & 29 \\
\hline & 4 & 1 & 5 & 2 & 3 \\
\hline \multirow[t]{2}{*}{ Honduras } & 1 & 10 & 19 & 18 & 33 \\
\hline & 4 & 4 & 3 & 10 & 9 \\
\hline \multirow[t]{2}{*}{ México } & 1 & 4 & - & - & 16 \\
\hline & 4 & 0 & - & - & 0 \\
\hline \multirow[t]{2}{*}{ Nicaragua } & 1 & 9 & 22 & 24 & 35 \\
\hline & 4 & 0 & 6 & 12 & 18 \\
\hline \multirow[t]{2}{*}{ Panamá } & 1 & 1 & 12 & 8 & 12 \\
\hline & 4 & 0 & 1 & 2 & 2 \\
\hline \multirow[t]{2}{*}{ Paraguay } & 1 & 7 & 17 & 19 & 23 \\
\hline & 4 & 0 & 3 & 4 & 10 \\
\hline \multirow[t]{2}{*}{ Uruguay } & 1 & 2 & 15 & 8 & 13 \\
\hline & 4 & 0 & 0 & 0 & 0 \\
\hline \multirow[t]{2}{*}{ Venezuela } & 1 & 5 & 16 & 15 & 25 \\
\hline & 4 & 1 & 2 & 2 & 8 \\
\hline
\end{tabular}

Fuente: Referencia 24

formal ha habido mejoras en la cobertura de la población desde 1985, pero estos adelantos han sido menores que en otras regiones del mundo: en 1995, apenas dos tercios de la población de ALC en edad escolar terminaba el cuarto grado de educación básica. La región del Sudeste Asiático, que tenía niveles educacionales formales similares en 1985, ahora ha sobrepasado a nuestra Región en la proporción de población matriculada en educación primaria y secundaria (25). El cuadro 2 muestra que en algunos países hasta un $10 \%$ de los niños procedentes de hogares del cuartil más pobre no ingresan a tiempo en la escuela primaria, en comparación con tan solo un $1 \%$ de los niños más ricos. De estos niños más pobres, el 20\%, y hasta un $44 \%$ en Brasil, se rezaga en los dos primeros años, frente a un $3 \%$ de los niños de familias más pudientes. Entre uno y dos tercios de los niños más pobres de algunos países no llegan a completar la enseñanza primaria, lo cual tiene una incidencia directa sobre la reproducción de la pobreza y un impacto muy directo sobre las condiciones de salud y desarrollo de la población en su conjunto.

El "Informe de desarrollo social" publicado recientemente por el BID recalcó la importancia de aumentar el acceso de los niños pobres a la instrucción formal como la intervención principal para re- ducir la pobreza y las desigualdades en la Región (26). Esta preocupación ha sido abordada también en varios estudios específicos.

Un estudio nacional realizado en Brasil en 1996 reveló que las tasas de bajo peso para la edad en los niños menores de 5 años se corresponden mayormente con el nivel de instrucción formal de la familia. Las tasas varían de un 19,3\% cuando el jefe de familia no tiene ninguna instrucción formal a solo un 3,4\% cuando tiene 11 o más años de instrucción, un aumento de casi seis veces. Las medidas intermedias de instrucción formal siguen el patrón correspondiente, con una tasa de bajo peso para la edad del 13,7\% con 1 a 3 años de instrucción formal, del $8,0 \%$ con 4 a 7 años y del 6,3\% con 8 a 10 años. Esto indica que cualquier continuación de la instrucción formal puede tener un efecto en la salud. Cuando se analiza el nivel educacional formal de la madre surgen patrones similares, tanto para las tasas de bajo peso para la edad, que van del 19,9\% con 0 a 3 años de instrucción formal al 3,3\% con 11 años o más, como para las tasas de bajo peso para el tamaño, que van del $24 \%$ con 0 a 3 años de escolaridad al 7\% con 6 o más años (27).

- Estudios realizados en Chile encontraron una relación entre la instrucción formal de las mujeres 
y la salud de sus hijos. Las tasas de mortalidad neonatal durante el período 1990-1995, estratificadas por nivel de instrucción formal de la madre, variaron de 13,5 por 1000 nacidos vivos para aquellas sin instrucción formal a 6 por 1000 para aquellas con 13 años o más. Las tasas de mortalidad posneonatales desciendieron de 24,5 por 1000 nacidos vivos a 2,6 para las mismas categorías, mostrando una diferencia de diez veces más cuando la madre es analfabeta que cuando tiene 13 o más años de escolaridad.

- Uno de los ejemplos más notables de la necesidad de estratificar las estadísticas nacionales de salud por grupos socioeconómicos para determinar la forma como se están captando los beneficios se ilustra por el aumento de la esperanza de vida de las mujeres chilenas entre la mitad de los años 80 y la mitad de los 90 . En Chile, las mujeres ganaron casi 2 años en su esperanza de vida a los 20 años de edad, pero las mujeres con 13 años o más de instrucción formal disfrutaron de casi todos los beneficios, ganando casi 10 años en una década, mientras que la ganancia en los grupos con niveles más bajos de instrucción fue insignificante (8).

\section{POBREZA Y ACCESO A LOS SERVICIOS BÁSICOS DE SALUD}

Unos pocos estudios por país, a nivel regional y local, han abordado las disparidades en los resultados de salud y el acceso a la atención entre las poblaciones con diferentes ingresos. En los últimos años, un conjunto de estudios ha permitido verificar la fuerte capacidad del factor distribución de los ingresos, ${ }^{2}$ no solo por el impacto absoluto de los ingresos, sino por su peso relativo en cada sociedad. El desdoblamiento de diferentes indicadores sociales por nivel de ingresos ha permitido evidenciar la existencia de fuertes desigualdades escondidas detrás de los indicadores globales o nacionales. La OPS ha coordinado una serie de estudios de casos sobre las desigualdades del sistema de salud en Brasil, Ecuador, Guatemala, Jamaica, México y Perú, que abarcan más de dos tercios de la población, del PIB y del gasto general de salud nacional del conjunto de los 45 países y territorios de ALC. En estos estudios se exploró la capacidad de las diferencias en organización, prestación y financiamiento de los sistemas de atención de salud nacionales para explicar

\footnotetext{
2 Esta variable, que suele medirse por quintil o decil de ingresos, sirve para evaluar el nivel de equidad en la distribución del ingreso y puede medirse a través de variables como el índice de Gini, la curva de Lorenz o el coeficiente 20/20, lo cual permite comparar países o regiones entre sí, o consigo mismos a lo largo del tiempo.
}

las desigualdades en los sistemas de salud y la respuesta de los diferentes sistemas nacionales a las necesidades de los pobres (28).

Se comprobó que las diferencias de los ingresos per cápita en estos países, expresados en USD ajustados para la paridad de poder adquisitivo, varían de casi USD 4000 en Guatemala a cerca de USD 6300 en Brasil y USD 7600 en México. Brasil y Guatemala son los países con el grado más alto de desigualdad de ingresos; el coeficiente de Gini para estos dos países es de alrededor de 0,60, mientras que la razón entre los quintiles más alto y más bajo de la distribución de los ingresos es de 47 en Brasil y 32 en Guatemala. Jamaica tiene el nivel más bajo de desigualdad de ingresos, con un coeficiente de Gini de 41 y una razón entre los quintiles más alto y más bajo de la distribución de los ingresos de 8 . La población que vive por debajo del marco de pobreza basado en el consumo - definido como aquellos cuyos ingresos están por debajo del costo de una canasta básica de productos alimenticios que proporcionan una ingestión o consumo mínimo de calorías y proteínasvaría de más de $50 \%$ en Ecuador, Perú y Guatemala a un $34 \%$ en Jamaica, $17 \%$ en Brasil y $10 \%$ en México. En Jamaica, los ingresos del 34\% de la población están por debajo del umbral de pobreza.

El estudio concluyó que, a pesar de la diversidad de condiciones socioeconómicas y una variedad de sistemas organizativos, financieros y de entrega, surgieron algunos patrones comunes, sobre todo en lo que respecta a la distribución de los beneficios del gasto público, el cual hace poco para corregir las desigualdades derivadas del gasto privado en salud y de la desigualdad de los ingresos. En Ecuador, Guatemala, Jamaica y Perú se analizó la distribución del gasto público en bienes y servicios de salud por quintiles de ingresos (cuadro 3).

Este cuadro muestra que, de los cuatro países, solo en Jamaica los beneficios del gasto público se distribuyen con preferencia a los grupos de menores ingresos; en este caso el 25,3\% del gasto público se destina al $20 \%$ más pobre de la población y solo el 15,2\% al quintil más rico. En los otros tres países esta distribución es neutral, como el caso de Perú, o está sesgada hacia los grupos de mayores ingresos, como ocurre en Guatemala y Ecuador. En estos dos últimos países, más del 30\% del gasto público se destina al quintil más rico de la población, una evidente inequidad, dadas las grandes desigualdades existentes en los tres últimos países en lo que respecta a la distribución de los ingresos y a otros parámetros de satisfacción de las necesidades básicas.

Se puede comprender entonces la preocupación por el incremento y la profundización de la calidad y cobertura de servicios, la necesidad de que lleguen a quienes más los necesitan y lo hagan de forma tal que puedan compensar, aunque solo sea 
CUADRO 3. Distribución porcentual de los beneficios del gasto público en salud, por quintil de ingresos. Ecuador, Guatemala, Jamaica y Perú, alrededor de 1998

\begin{tabular}{lccccc}
\hline & \multicolumn{5}{c}{ Quintil de ingresos } \\
\cline { 2 - 6 } \multicolumn{1}{c}{ País } & 1 & 2 & 3 & 4 & 5 \\
\hline Ecuador & 12,5 & 15,0 & 19,4 & 22,5 & 30,5 \\
Guatemala & 12,8 & 12,7 & 16,9 & 26,3 & 31,3 \\
Jamaica & 25,3 & 23,9 & 19,4 & 16,2 & 15,2 \\
Perú & 20,1 & 20,7 & 21,0 & 20,7 & 17,5 \\
\hline
\end{tabular}

Fuente: Referencia 28.

en parte, las desventajas que producen el nivel de ingresos y la marginación. En síntesis, la pobreza es un fenómeno multidimensional que se reproduce de forma intergeneracional y que ha crecido de manera absoluta en la última década en la Región, pese al moderado y sostenido crecimiento económico que la mayor parte de los países experimentaron durante la década de los noventa. La asignación de recursos y la distribución de bienes y servicios, tanto públicos como privados, aún tienden a con- centrarse en los estratos socioeconómicos medios y altos, reforzando los fenómenos de deprivación y exclusión social.

El cuadro 4 muestra cómo siguen reproduciéndose en los países las diferencias según el nivel de ingresos en el acceso a un servicio básico como la disponibilidad de agua corriente. Por ejemplo, en el caso de Bolivia se constata que en la población urbana el decil inferior de ingresos se encuentra cubierto en un $82,1 \%$, mientras que en el decil superior alcanza al 98,1\% de los hogares. Los países de menores ingresos, como Ecuador, El Salvador, Nicaragua, Paraguay y Perú, o con mayores inequidades, como Brasil y Panamá, siguen el mismo patrón. Países como Chile, Colombia y Jamaica muestran muy pocas diferencias en la población urbana, ya que comienzan en su decil más bajo con valores superiores al $90 \%$, pero muy grandes en la población rural.

La correlación entre disponibilidad de agua corriente y nivel de ingresos es mucho más marcada en la población urbana, que además presenta, en conjunto, una cobertura mayor que las poblaciones rurales, que a su vez se diferencian en algunos

CUADRO 4. Porcentaje de hogares con agua corriente, por deciles (primero y décimo) de ingresos y área geográfica de asentamiento. Once países de América Latina y el Caribe, alrededor de 1999

\begin{tabular}{|c|c|c|c|c|}
\hline \multirow[b]{2}{*}{ País } & & \multicolumn{3}{|c|}{ Porcentaje de hogares con agua corriente } \\
\hline & & Decil 1 & Decil 10 & Población total \\
\hline \multirow[t]{2}{*}{ Bolivia } & Urbano & 82,1 & 98,1 & 90,6 \\
\hline & Rural & 14,3 & 41,5 & 23,0 \\
\hline \multirow[t]{2}{*}{ Brasil } & Urbano & 53,5 & 97,3 & 89,6 \\
\hline & Rural & 2,6 & 32,3 & 19,3 \\
\hline \multirow[t]{2}{*}{ Chile } & Urbano & 96,8 & 99,7 & 98,8 \\
\hline & Rural & 27,7 & 43,1 & 36,0 \\
\hline \multirow[t]{3}{*}{ Colombia } & Urbano & 91,1 & 99,2 & 97,4 \\
\hline & Rurala & 71,4 & 91,8 & 78,2 \\
\hline & Rural $^{b}$ & 29,6 & 41,0 & 31,2 \\
\hline \multirow[t]{3}{*}{ Ecuador } & Urbano & 56,2 & 90,8 & 75,3 \\
\hline & Rurala $^{a}$ & 42,3 & 49,4 & 46,3 \\
\hline & Rural $^{b}$ & 11,2 & 26,3 & 18,5 \\
\hline \multirow[t]{2}{*}{ El Salvador } & Urbano & 39,3 & 88,8 & 70,5 \\
\hline & Rural & 16,2 & 39,6 & 25,5 \\
\hline \multirow[t]{3}{*}{ Jamaica } & Kingston & 95,7 & 100,0 & 97,4 \\
\hline & Urbano & 62,7 & 89,5 & 79,4 \\
\hline & Rural & 23,2 & 54,8 & 38,8 \\
\hline \multirow[t]{2}{*}{ Nicaragua } & Urbano & 58,3 & 96,4 & 83,9 \\
\hline & Rural & 7,3 & 53,3 & 30,5 \\
\hline \multirow[t]{4}{*}{ Panamá } & Urbano & 84,0 & 100,0 & 95,4 \\
\hline & Rural & 55,8 & 92,8 & 79,9 \\
\hline & Ruralc & 16,7 & 45,5 & 24,4 \\
\hline & Indígena & 39,0 & 34,4 & 37,1 \\
\hline \multirow[t]{2}{*}{ Paraguay } & Urbano & 35,0 & 87,7 & 66,9 \\
\hline & Rural & 1,8 & 30,6 & 13,3 \\
\hline \multirow[t]{2}{*}{ Perú } & Urbano & 57,7 & 97,0 & 85,0 \\
\hline & Rural & 35,0 & 34,4 & 41,9 \\
\hline
\end{tabular}


países en función de su facilidad de acceso. Estas diferencias son muy relevantes para determinar la salud infantil, por ejemplo, ya que la probabilidad de tener episodios diarreicos en menores de 5 años - una de las principales causas de mortalidad infantil tardía y desnutrición- está en razón inversa de la disponibilidad de agua corriente en el domicilio; este es uno de los principales factores protectores, superado solo por el nivel de educación de la madre, lo cual bien podría ser una variable colineal con la disponibilidad de agua corriente (29).

\section{POBREZA, INGRESOS, NUTRICIÓN $Y$ ACCESO A SERVICIOS DE SALUD INFANTIL Y REPRODUCTIVA}

En el caso de la desnutrición se constata de nuevo que la relación entre este indicador y el nivel de ingresos es muy robusta. En el cuadro 5, basado en los análisis de las Encuestas de Condiciones de Vida de nueves países de la Región (30), se puede observar que la tasa de desnutrición de los niños del quintil de ingresos más alto es, según los países, entre 3,5 y 10 veces menor que la del quintil inferior. Además la tasa de desnutrición guarda en todos los países seleccionados una relación en gradiente a lo largo de la escala del nivel de ingresos, de forma tan proporcional que semeja una relación dosis-respuesta.

En lo que respecta al acceso a la atención médica básica para la población infantil, como el tratamiento y prevención de infecciones respiratorias agudas, se observan los mismos gradientes y disparidades por nivel de ingresos. Los niños más pobres tienen entre la tercera parte y la mitad de las posibilidades de recibir tratamiento oportuno y eficaz, en comparación con los más ricos (cuadro 6).

Ni siquiera un servicio tan básico y de tan amplia cobertura en la Región como la vacunación se escapa de este patrón (cuadro 7). Nótese, sin embargo, que en algunos países el quintil de mayores ingresos, que tiende a ser atendido en el subsector privado, presenta una cobertura inferior a la del cuarto, y a veces a la del tercer quintil, lo cual amerita ser contemplado por las estrategias de eliminación de enfermedades inmunoprevenibles en la Región. La razón entre los grupos de mayor y menor ingreso oscila entre 0,38 en Paraguay y prácticamente la unidad en el caso de Guatemala.

Teniendo en cuenta lo anterior, no es sorprendente que la TMI también muestre claras y escalonadas diferencias por quintil de ingresos (cuadro 8). La sobremortalidad infantil de los más pobres oscila entre 1,3 veces en Haití y más de 4 veces en Bolivia y Perú.

Una relación muy similar ocurre entre el nivel de ingresos y los porcentajes de mujeres embarazadas con atención prenatal y de partos atendidos por profesionales (cuadro 9). Con la excepción de la República Dominicana, se observan diferencias realmente alarmantes en la baja cobertura de los grupos más pobres: las mujeres de menores ingresos que reciben atención profesional en el momento del parto son menos del $20 \%$ y entre la tercera parte y la mitad de ellas no reciben atención prenatal durante la gestación. Evidentemente, esta situación tiene implicaciones muy graves para la calidad en que se puede desarrollar el período prenatal y la primera infancia de los niños más pobres, y su consiguiente impacto en el desarrollo psicosocial, cognitivo y físico en años posteriores de la vida.

Vale la pena señalar que otra dimensión importante de la salud reproductiva, la utilización de métodos anticonceptivos modernos, también muestra una distribución diferenciada y en gradiente por el quintil de ingresos (cuadro 10), con lo que este factor se suma a los anteriores para potenciar el riesgo de mortalidad materna y neonatal en los grupos de menores ingresos.

CUADRO 5. Tasa de desnutrición en niños, por quintil de ingresos. Nueve países de América Latina y el Caribe, alrededor de 1999

\begin{tabular}{lrrrrrr}
\hline \multirow{7}{*}{ País } & \multicolumn{5}{c}{ Quintil de ingresos } & Razón entre los \\
\cline { 2 - 5 } & 1 & 2 & \multicolumn{1}{c}{3} & \multicolumn{1}{c}{4} & 5 & quintiles 1 y 5 \\
\hline Bolivia & 39,2 & 29,0 & 22,3 & 11,1 & 6,0 & 6,53 \\
Brasil & 23,2 & 8,7 & 5,0 & 3,9 & 2,3 & 10,09 \\
Colombia & 23,7 & 16,7 & 13,4 & 7,7 & 5,9 & 4,02 \\
Guatemala & 64,6 & 61,6 & 53,5 & 33,5 & 12,1 & 5,34 \\
Haití & 45,5 & 33,0 & 32,3 & 25,2 & 12,8 & 3,56 \\
Nicaragua & 38,1 & 29,1 & 22,7 & 13,0 & 8,3 & 4,59 \\
Paraguay & 22,5 & 19,0 & 12,5 & 6,3 & 3,0 & 7,5 \\
Perú & 45,6 & 30,8 & 18,8 & 9,9 & 5,2 & 8,77 \\
R. Dominicana & 21,5 & 10,3 & 7,8 & 5,6 & 2,5 & 8,60 \\
\hline
\end{tabular}


CUADRO 6. Porcentaje de niños que recibieron tratamiento por infecciones respiratorias, por quintil de ingresos. Ocho países de América Latina y el Caribe, alrededor de 1999

\begin{tabular}{lrrrrrr}
\hline \multicolumn{1}{c}{ País } & \multicolumn{9}{c}{ Quintil de ingresos } & Razón entre los \\
\cline { 2 - 5 } quintiles 1 y 5
\end{tabular}

Fuente: Referencia 30.

CUADRO 7. Cobertura de vacunaciones completa, por quintil de ingreso. Nueve países de América Latina y el Caribe, alrededor de 1999

\begin{tabular}{lcccccc}
\hline \multirow{2}{*}{ País } & \multicolumn{5}{c}{ Quintil de ingresos } & Razón entre los \\
\cline { 2 - 5 } & 1 & 2 & 3 & 4 & 5 & quintiles 1 y 5 \\
\hline Bolivia & 21,8 & 24,9 & 21,0 & 33,4 & 30,6 & 0,712 \\
Brasil & 56,6 & 74,0 & 84,9 & 83,1 & 73,8 & 0,767 \\
Colombia & 53,8 & 66,9 & 68,1 & 70,6 & 74,1 & 0,726 \\
Guatemala & 41,2 & 43,0 & 47,1 & 38,3 & 42,5 & 0,969 \\
Haití & 18,8 & 20,1 & 35,3 & 37,9 & 44,1 & 0,426 \\
Nicaragua & 61,0 & 74,6 & 75,3 & 85,7 & 73,1 & 0,834 \\
Paraguay & 20,2 & 30,8 & 36,4 & 40,7 & 53,0 & 0,381 \\
Perú & 55,3 & 63,8 & 63,5 & 71,7 & 66,0 & 0,838 \\
R. Dominicana & 28,0 & 30,2 & 46,9 & 42,6 & 51,7 & 0,542 \\
\hline
\end{tabular}

Fuente: Referencia 30.

CUADRO 8. Tasa de mortalidad infantil, por quintil de ingresos. Nueve países de América Latina y el Caribe, alrededor de 1999

\begin{tabular}{lrrrrrr}
\hline & \multicolumn{5}{c}{ Quintil de ingresos } & Razón entre los \\
\cline { 2 - 5 } \multicolumn{1}{c}{ País } & 1 & 2 & 3 & 4 & 5 & quintiles 1 y 5 \\
\hline Bolivia & 106,5 & 85,0 & 75,5 & 38,6 & 25,5 & 4,176 \\
Brasil & 83,2 & 46,7 & 32,9 & 24,7 & 28,6 & 2,909 \\
Colombia & 40,8 & 31,4 & 27,0 & 31,5 & 16,2 & 2,519 \\
Guatemala & 56,9 & 79,7 & 55,7 & 46,7 & 35,0 & 1,626 \\
Haití & 93,7 & 93,6 & 85,6 & 81,7 & 74,3 & 1,261 \\
Nicaragua & 50,7 & 53,7 & 45,7 & 40,2 & 25,8 & 1,965 \\
Paraguay & 42,9 & 36,5 & 46,1 & 33,5 & 15,7 & 2,732 \\
Perú & 78,3 & 53,6 & 34,4 & 36,0 & 19,5 & 4,015 \\
R. Dominicana & 66,7 & 54,5 & 52,3 & 33,5 & 23,4 & 2,850 \\
\hline
\end{tabular}

Fuente: Referencia 30.

\section{GOBERNABILIDAD, EXCLUSIÓN SOCIAL Y SALUD}

En este contexto importa reconocer no solo la importancia de las fuertes relaciones entre la salud y la pobreza, sino también que el sector de la salud puede hacer mucho para revertir esta si- tuación. La idea es implementar políticas activas que protejan selectivamente a la población, compensando otras inequidades $\mathrm{y}$, por un sentido de coherencia, puedan remover todos aquellos mecanismos que se sumen a los de exclusión o asignación inequitativa de recursos. Las desigualdades en la distribución de los ingresos están muy asociadas 
CUADRO 9. Porcentaje de mujeres embarazadas con atención prenatal y de partos atendidos por profesionales, por quintil de ingresos. Nueve países de América Latina y el Caribe, alrededor de 1999

\begin{tabular}{|c|c|c|c|c|c|c|c|}
\hline \multirow[b]{2}{*}{ País } & \multicolumn{5}{|c|}{ Quintil de ingresos } & \multirow{2}{*}{$\begin{array}{l}\text { Promedio } \\
\text { poblacional }\end{array}$} & \multirow{2}{*}{$\begin{array}{c}\text { Razón entre los } \\
\text { quintiles } 1 \text { y } 5\end{array}$} \\
\hline & 1 & 2 & 3 & 4 & 5 & & \\
\hline \multicolumn{8}{|l|}{ Bolivia } \\
\hline Atención prenatal & 38,8 & 57,8 & 70,4 & 88,6 & 95,3 & 65,1 & 0,407 \\
\hline Atención profesional al parto & 19,8 & 44,8 & 67,7 & 87,9 & 97,9 & 56,7 & 0,202 \\
\hline \multicolumn{8}{|l|}{ Brasil } \\
\hline Atención prenatal & 67,5 & 87,7 & 93,4 & 96,9 & 98,1 & 85,6 & 0,688 \\
\hline Atención profesional al parto & 71,6 & 88,7 & 95,7 & 97,7 & 98,6 & 87,7 & 0,726 \\
\hline \multicolumn{8}{|l|}{ Colombia } \\
\hline Atención prenatal & 67,5 & 87,7 & 93,4 & 96,9 & 98,1 & 85,6 & 0,688 \\
\hline Atención profesional al parto & 60,6 & 85,2 & 92,8 & 98,9 & 98,1 & 84,5 & 0,618 \\
\hline \multicolumn{8}{|l|}{ Guatemala } \\
\hline Atención prenatal & 34,6 & 41,1 & 49,3 & 72,2 & 90,0 & 52,5 & 0,384 \\
\hline Atención profesional al parto & 9,3 & 16,1 & 31,1 & 62,8 & 91,5 & 34,8 & 0,102 \\
\hline \multicolumn{8}{|l|}{ Haití } \\
\hline Atención prenatal & 44,3 & 60,0 & 72,3 & 83,7 & 91,0 & 67,7 & 0,487 \\
\hline Atención profesional al parto & 24,0 & 37,3 & 47,4 & 60,7 & 78,2 & 46,3 & 0,307 \\
\hline \multicolumn{8}{|l|}{ Nicaragua } \\
\hline Atención prenatal & 67,0 & 80,9 & 86,9 & 89,0 & 96,0 & 81,5 & 0,698 \\
\hline Atención profesional al parto & 32,9 & 58,8 & 79,8 & 86,0 & 92,3 & 64,6 & 0,356 \\
\hline \multicolumn{8}{|l|}{ Paraguay } \\
\hline Atención prenatal & 69,5 & 79,5 & 85,6 & 94,8 & 98,5 & 83,9 & 0,706 \\
\hline Atención profesional al parto & 41,2 & 49,9 & 69,0 & 87,9 & 98,1 & 66,0 & 0,420 \\
\hline \multicolumn{8}{|l|}{ Perú } \\
\hline Atención prenatal & 37,3 & 64,8 & 79,1 & 87,7 & 96,0 & 67,3 & 0,389 \\
\hline Atención profesional al parto & 13,7 & 48,0 & 75,1 & 90,3 & 96,6 & 56,4 & 0,142 \\
\hline \multicolumn{8}{|l|}{ República Dominicana } \\
\hline Atención prenatal & 96,1 & 98,2 & 99,0 & 99,2 & 99,9 & 98,3 & 0,962 \\
\hline Atención profesional al parto & 88,6 & 96,9 & 97,3 & 98,4 & 97,8 & 95,3 & 0,906 \\
\hline
\end{tabular}

Fuente: Referencia 30.

a disparidades en el acceso a los servicios de salud y a otros servicios sociales relevantes para los niveles de salud de la población y la morbimortalidad. La relación sistemática entre mortalidad, estatus económico e inequidad ha comenzado a llamar la atención de una nueva generación de investigadores que han comprobado que la diferencia de ingresos suma factores de riesgo para la salud y para la mortalidad prematura, y que tiene una potencia explicativa aún mayor que la del valor absoluto del ingreso. Esto ha reforzado la valoración del capital social como un factor relevante para la protección de la salud (31).

Sin lugar a dudas, la estabilidad de los regímenes democráticos es uno de los logros más importantes de las últimas dos décadas en la Región. Sin embargo queda mucho por hacer en la construcción de procesos de participación ciudadana, en el mejoramiento de la credibilidad de las instituciones y en la lucha contra la corrupción. La globalización tensiona las bases de la gobernabilidad debido a que, por un lado, las presiones ejercidas por las fuerzas supranacionales y transnacionales erosionan la soberanía y debilitan la identidad y naturaleza del Estado en la medida en que intervienen en los asuntos nacionales y presionan para el estableci- miento de leyes universales. Por otro lado, la globalización demanda una redefinición de los conocimientos, actitudes y habilidades requeridos de los líderes políticos y del servicio civil. En otras palabras, la globalización impone la necesidad de reinventar el liderazgo y la sociedad civil, y de reformar el Estado (32).

De igual manera, la globalización y la revolución tecnológica que la acompaña plantean muchos retos para los procesos democráticos latinoamericanos debido a que, si bien es cierto que multiplican el número de actores políticos, los distintos sectores continúan desigualmente representados en el caso de ALC. Otro efecto secundario de la globalización y la revolución tecnológica es que permiten a una pequeña minoría incrementar sus ingresos de manera significativa, mientras que la gran mayoría lucha por mantener sus ingresos estables, y otros muchos los ven disminuir considerablemente, lo cual causa gran inseguridad. Debemos agregar el riesgo que representa el incremento de la pobreza estructural, la creciente marginalización de grandes sectores de la población y la desarticulación de la fuerza laboral (33).

La exclusión social es sin duda alguna el mayor riesgo que acarrea la globalización. Un gran 
CUADRO 10. Porcentaje de mujeres en edad reproductiva que utilizan modernos métodos anticonceptivos, por quintil de ingresos. Nueve países de América Latina y el Caribe, alrededor de 1999

\begin{tabular}{lrrrrrr}
\hline \multirow{7}{r}{ País } & \multicolumn{5}{c}{ Quintil de ingresos } & \multirow{2}{*}{$\begin{array}{c}\text { Razón entre los } \\
\text { quintiles 1 y 5 }\end{array}$} \\
\cline { 2 - 5 } & 1 & 2 & 3 & 4 & 5 & 0,156 \\
Bolivia & 7,1 & 17,2 & 22,2 & 32,2 & 45,6 & 0,727 \\
Brasil & 55,8 & 68,9 & 73,6 & 73,8 & 76,8 & 0,642 \\
Colombia & 42,2 & 59,6 & 62,7 & 64,2 & 65,7 & 0,095 \\
Guatemala & 5,4 & 10,1 & 21,4 & 37,4 & 57,1 & 0,234 \\
Haití & 4,9 & 7,4 & 12,7 & 20,4 & 20,9 & 0,626 \\
Nicaragua & 40,2 & 55,5 & 60,3 & 65,4 & 64,2 & 0,447 \\
Paraguay & 20,6 & 25,3 & 34,4 & 44,3 & 46,1 & 0,477 \\
Perú & 24,0 & 37,5 & 45,2 & 48,9 & 50,3 & 0,804 \\
R. Dominicana & 51,2 & 61,7 & 58,2 & 61,5 & 63,7 & \\
\hline
\end{tabular}

Fuente: Referencia 30.

número de personas pierde primero su puesto de trabajo y posteriormente la participación política y social en su comunidad. La disminución de la participación ciudadana en las actividades de la comunidad y la pérdida de la confianza entre los miembros de la comunidad tiene un grave impacto en el capital social. Al contrario que el capital humano, o capital físico, el capital social es un bien público creado como subproducto de las relaciones sociales. Se refiere a "los rasgos de las organizaciones sociales, tales como confianza, normas y redes que pueden mejorar la eficiencia de la sociedad facilitando la coordinación de acciones" (34). La brecha entre los ricos y los pobres es grande y está vinculada negativamente al nivel de inversión en el capital social. Es decir, la disminución de la inversión en el capital social es una vía por medio de la cual las crecientes inequidades en el nivel de ingresos ejercen su efecto en el índice de mortalidad (35).

El esfuerzo para lograr una mejor salud en el desarrollo humano sostenible debe incluir políticas conducentes al crecimiento económico sostenido con una distribución de los ingresos más equitativa, vinculadas con intervenciones que mejoren las capacidades esenciales de los pobres (servicios de salud; ambientes saludables, particularmente el acceso a servicios adecuados de agua y saneamiento; nutrición, y oportunidades educativas). En consecuencia, una de las funciones esenciales de la gestión en salud pública es vigilar, tomar medidas y abogar por la seguridad del ambiente físico y social, y velar por que toda actividad de desarrollo se evalúe adecuadamente en cuanto a sus repercusiones ambientales y sociales en la salud de la población, y en particular de sus miembros más vulnerables y desamparados.

El sector de la salud está llamado a cumplir un conjunto de funciones esenciales de promoción y de protección de la salud de la población, ya que la ciudadanía tiene un derecho inalienable a recibir estas garantías y servicios como participantes en su sociedad. Para el ejercicio efectivo de este rol de promoción y protección de la salud por parte del Estado, tanto a nivel del gobierno central como del gobierno local, es indispensable el desarrollo y fortalecimiento de un marco legal, normativo y regulador adecuado y actualizado, mediante el cual se facilite la colaboración efectiva entre los sectores público y privado, y de estos con la sociedad civil, para proteger la salud colectiva, sobre todo la de los sectores más vulnerables y desprotegidos.

\section{SYNOPSIS}

\section{Health, human development, and governance in Latin America and the Caribbean at the beginning of the 21 st century}

The issue of the reciprocal relationship between health and development has recently taken on greater importance in Latin America and the Caribbean (LAC), given the persistence of extreme poverty and the political and social difficulties due to macroeconomic imbalances and crises of governance. This piece reviews concepts of sustainable human development, social determinants of health in general and of health inequities in particular (gender, ethnic group, income level), and the relationship between health and economic growth in the medium term and the long term. An analysis is made of how persistent poverty in countries of LAC relates to disparities in health conditions, access to health services, and health care financing, as well as to such health determinants as nutrition and environmental sanitation. Health inequities most strongly affect the most excluded and vulnerable sectors of the population. In the face of this situation, the author stresses that putting a priority on health inequities is vital to safeguarding the governability and the social and political stability of countries in LAC in the next decade. 


\section{REFERENCIAS}

1. Organización Panamericana de la Salud. Salud, agua potable y saneamiento en el desarrollo humano sostenible. Washington, D.C.: OPS; 2001. (Documento CD43/10).

2. Wing SB, Richardson D. Material living conditions and health in the United States, Canada and Western Europe: review of recent literature and bibliography. Washington, D.C.: Pan American Health Organization; 1999. (Serie: Research in public health, No. 9).

3. Bambas A, Casas JA. Assessing equity in health: conceptual criteria. En: Equity and health: views from the Pan American Sanitary Bureau. Washington DC: PAHO; 2001. Pp. 12-21.

4. Casas JA, Dachs NW, Bambas A. Health disparities in Latin America and the $\mathrm{Ca}$ ribbean: The role of social and economic determinants. En: Equity and health: views from the Pan American Sanitary Bureau. Washington DC: PAHO; 2001. Pp. 22-49.

5. Merryfield A, Swyngedouw E. The urbanization of injustice. New York: University Press; 1997.

6. Organización Panamericana de la Salud. Informe sobre el proyecto multicéntrico: la salud y los ancianos. Documento presentado en la Reunion del Comité Asesor de Investigaciones en Salud, Washington, D.C., 16-18 julio 1997. (OPS/ CAIS/97.09).

7. Inter-American Development Bank Economic and social progress in Latin America, 1998-1999 report: facing up to inequality in Latin America. Washington, D.C.; IADB; 1999.

8. Vega J, Hollstein RD, Delgado I, Marshall G, Yach D. Social inequalities and health in an intermediate-development nation: eduacation and adult mortality in Chile, 1980-1996. En: Evans T, Whitehead M, Diderichsen F, Bhuiya A, Wirth $\mathrm{M}$, eds. Challenging inequities in health: from ethics to action. New York: Oxford University Press; 2001. Pp. 123-137.

9. Bloom DE, Canning D, Sevilla J. The effect of health on economic growth: theory and evidence. Cambridge, $\mathrm{MA}: \mathrm{Na}-$ tional Bureau of Economic Research; 2001. (Working Paper No.w8587).

10. Wilkinson RG. Unhealthy societies: the afflictions of inequality. London: Routledge; 1996.

11. Sen, $B$. The life and death question: health as a contributor to development, draft, WHO, June 2000. En Partnerships in health and poverty: towards a common agenda. Disponible en: http:// www.sidint.org/programmes/he-
alth/Reportpartnershipinhealth.PDF. P. 9.

12. Keating DP, Hertzman C. Developmental health and the wealth of nations: social, biological and educational dynamics. New York: Guilford Press; 2000

13. Deutsch R. How early childhood intervention can reduce inequality: an overview of recent findings. Washington, D.C.: Inter-American Development Bank; 1998. (2/99, POV-105, E). Disponible en: http://www.iadb.org/sds/ doc/923eng.pdf

14. Van der Gaag J, Jee-Pan T. The benefits of early child development programs: an economic analysis. Washington, D.C.: World Bank; 1998.

15. Smith GD, Hart C, Blane D, Hole D. Adverse socioeconomic conditions in childhood and cause specific adult mortality: prospective observational study. BMJ 1998;316:1631-1635.

16. Strauss J, Duncan T. Health, nutrition and economic development. J Econ Lit 1998;36:766-817.

17. Gallup JL, Sachs JD, Mellinger A. Geography and economic development. Presented at the Annual Bank Conference on Development Economics, World Bank. April, 1998. Disponible en: $\begin{array}{llll}\mathrm{h} & \mathrm{t} & \mathrm{t} & \mathrm{p} \\ \text { www2.cid.harvard.edu/cidwp/001.pdf. }\end{array}$

18. Davedoff W, Schultz P (eds.) Wealth from health, linking social investments to earnings in Latin America. Washington, D.C.: Inter-American Development Bank; 2000.

19. Bloom DE, Sachs JD. Geography, demography and economic growth in Africa. Brookings Papers on Economic Activity, 1998;2:207-295. Disponible en: http:// www2.cid.harvard.edu/cidpapers/ brookafr.pdf

20. Pan American Health Organization/ Inter-American Development Bank/ World Bank/United Nations Development Program/United Nations Economic Commission for Latin America and the Caribbean. Investment in health: social and economic returns. Washington, D.C.: PAHO; 2001

21. Sen A. Development as freedom. New York: Random House; 1999.

22. Banco Mundial. Informe mundial de desarrollo, 2000. Washington, D.C.: Banco Mundial; 2000.

23. Kliksberg B. Los escenarios sociales en América Latina y el Caribe. Rev Panam Salud Publica 2000;8:105-111.

24. Comisión Económica para América Latina y el Caribe. La brecha de la equidad, 2000. Santiago de Chile: CEPAL; 2000.
25. World Bank. World development report, 1998. Washington, D.C.: World Bank; 1998.

26. Inter-American Development Bank. Social development report, 1998-99. Washington, D.C.: IADB; 1999.

27. Epidemiological Studies in Health $\mathrm{Nu}$ trition, University of São Paulo. Melhoria em indicadores de saúde associados a pobreza no Brasil dos anos 90 . University of São Paulo School of Public Health. (Internal Report).

28. Suárez-Berenguela RM. Health systems inequalities and inequities in Latin America and the Caribbean: findings and policy implications. En: Investment in health: social and economic returns. Washington D.C.: Pan American Health Organization; 2001. Pp. 119-142. (PAHO Scientific Publication No. 582)

29. Wagstaff A. Poverty, equity, and health: some research findings. En: Equity and health: views from the Pan American Sanitary Bureau. Washington, D.C.: Pan American Health Organization, 2001. (Occasional Publication No. 8).

30. Gwatkin DR, Pande R, Rutstein S. Socioeconomic differences in health, nutrition, and population in selected countries. Washington, D.C: World Bank; 2000. (HNP/Poverty Thematic Group)

31. Deaton A. Health, inequality, and economic development. Geneva: Commission on Macroeconomics and Health. 2001. (CMH Working Paper Series. Paper No. WG1:3) Disponible en: http:// www.cmhealth.org/docs/wg1_paper3. pdf

32. Prats J. Gobernabiliad y globalización los desafíos del desarrollo para después del 2000. En: Gobernabilidad y salud: políticas y participación social. Washington, D.C.: Organización Panamericana de la Sslud; 1999. Pp. 35-62.

33. Bezanson K. Desarrollo internacional en la víspera del tercer milenio. Conferencia magistral al IV Congreso Panamericano de Información en Ciencias de la Salud. San José, Costa Rica, 24-27 marzo 1998. En: Organización Panamericana de la Salud. Centro Latinoamericano y del Caribe de Información en Ciencias de la Salud. Biblioteca Virtual. São Paulo: BIREME; 1998. Pp. 36-62.

34. Putnam R. Making democracy work: civic traditions in modern Italy. Princeton: Princeton University Press; 1993. P. 167.

35. Kawachi I, Kennedy BP, Lochner K Prothrow-Stith D. Social capital, income inequality and mortality. Am J Public Health 1997;87:1491-1514. 